\title{
Phthalate-associated hypertension in premature infants: a prospective mechanistic cohort study
}

\author{
Randall Jenkins $^{1}$ (1) $\cdot$ Shane Tackitt ${ }^{2} \cdot$ Ladawna Gievers $^{1} \cdot$ Sandra Iragorri $^{1} \cdot$ Kylie Sage $^{3} \cdot$ Tonya Cornwall $^{1}$. \\ Declan O'Riordan ${ }^{4}$. Jennifer Merchant ${ }^{4} \cdot$ David Rozansky $^{1}$
}

Received: 20 December 2018 / Revised: 5 February 2019 / Accepted: 18 March 2019 / Published online: 26 April 2019

(C) The Author(s) 2019

\begin{abstract}
Background Phthalates are associated with increased blood pressure in children. Large exposures to di-(2-ethylhexyl) phthalate (DEHP) among premature infants have been a cause for concern.

Methods We conducted a prospective observational cohort study to determine if DEHP exposures are related to systolic blood pressure (SBP) in premature infants, and if this exposure is associated with activation of the mineralocorticoid receptor (MR). Infants were monitored longitudinally for 8 months from birth. Those who developed idiopathic hypertension were compared with normotensive infants for DEHP exposures. Appearance of urinary metabolites after exposure was documented. Linear regression evaluated the relationship between DEHP exposures and SBP index and whether urinary cortisol/cortisone ratio (a surrogate marker for $11 \beta$-HSD2 activity) mediated those relationships. Urinary exosomes were quantified for sodium transporter/channel expression and interrogated against SBP index.

Results Eighteen patients met the study criteria, nine developed transient idiopathic hypertension at a postmenstrual age of $40.6 \pm 3.4$ weeks. The presence of urinary DEHP metabolites was associated with prior IV and respiratory tubing DEHP exposures $(p<0.05)$. Both IV and respiratory DEHP exposures were greater in hypertensive infants $(p<0.05)$. SBP index was related to DEHP exposure from IV fluid $(p=0.018)$, but not respiratory DEHP. Urinary cortisol/cortisone ratio was related to IV DEHP and SBP index $(p<0.05)$. Sodium transporter/channel expression was also related to SBP index $(p<0.05)$.

Conclusions Increased blood pressure and hypertension in premature infants are associated with postnatal DEHP exposure. The mechanism of action appears to be activation of the MR through inhibition of $11 \beta-H S D 2$.
\end{abstract}

Keywords Hypertension $\cdot$ Neonatal $\cdot$ Phthalates $\cdot$ Blood pressure

\section{Abbreviations}

DEHP Di-(2-ethylhexyl) phthalate

Electronic supplementary material The online version of this article (https://doi.org/10.1007/s00467-019-04244-4) contains supplementary material, which is available to authorized users.

Randall Jenkins

jenkinra@ohsu.edu

1 Department of Pediatrics, Oregon Health \& Science University, 707 SW Gaines Road, Mail Code CDRC-P, Portland, OR 97239, USA

2 HCA Medical City Weatherford, Weatherford, TX, USA

3 Biostatistics and Design Program, School of Public Health, Oregon Health \& Science University, Portland, OR, USA

4 St. Luke's Regional Medical Center, Boise, ID, USA
PVC Polyvinyl chloride

IV Intravenous

NICU Newborn intensive care unit

MR Mineralocorticoid receptor

$11 \beta$-HSD2 $11 \beta$-Hydroxysteroid dehydrogenase 2

AME Apparent mineralocorticoid excess

MEHP Mono-(2-ethylhexyl) phthalate

SBP Systolic blood pressure

PMA Postmenstrual age

PRA Plasma renin activity

MEHHP Mono-(2-ethyl-5-hydroxyhexyl) phthalate

MEOHP Mono-(2-ethyl-5-oxohexyl) phthalate

$\mathrm{ENaC} \quad$ Epithelial $\mathrm{Na}+$ channel

pNCC Phosphorylated (activated)

$\mathrm{Na}+-\mathrm{Cl}^{-}$cotransporter 


\section{Introduction}

The only phthalate approved by the U.S. Food and Drug Administration for use in medical supplies is di-(2-ethylhexyl) phthalate (DEHP), a compound commonly added to polyvinyl chloride (PVC) to soften plastic. DEHP has been identified in a variety of hospital devices including a selection of intravenous (IV) fluid bags and some respiratory equipment [1-5]. Inherent use of such devices in a neonatal intensive care unit (NICU) may expose small premature infants to relatively high levels of DEHP, exceeding typical adult exposures by several orders of magnitude $[1,3,6]$.

In 2013, with a report from Trasande et al. and later corroborated by others, phthalates surfaced as a cause of increased blood pressure in children and adults [7-10]. At present, the mechanism of this increase remains unclear. However, only a few years earlier, Zhao et al. pointed toward a possible mechanism by showing that certain DEHP metabolites cause sodium retention due to a licorice-like action inhibiting the enzyme $11 \beta$-hydroxysteroid dehydrogenase type $2(11 \beta$ HSD2) [11].

In 2017, our group reported on a large cohort of 97 premature infants with idiopathic low-renin hypertension, some of whom had chronic lung disease [12]. Al Awad et al. recently reported on a homogenous group of premature infants with low-renin hypertension, all of whom had chronic lung disease [13]. Hypertensive patients in these two studies all shared certain similarities including near-universal low or undetectable plasma renin activity (PRA), presentation of high blood pressure around 35-40 weeks postmenstrual age (PMA), and an excellent response to treatment with spironolactone. These similarities suggest the possibility of a unifying mechanism for the observed hypertension involving sodium transport in the distal nephron.

When considered in concert, the three concepts outlined above - large exposure to DEHP, a potential mechanism of sodium retention tied to DEHP, and cohorts of premature infants with low-renin hypertension-provide a basis for the hypothesis that DEHP increases blood pressure and may cause hypertension in premature infants. This current report tests this hypothesis both by comparing DEHP exposure between hypertensive and normotensive premature infants, as well as by examining the linear relationship between DEHP exposure and blood pressure in the overall cohort. Further, we investigate the infants' absorption of DEHP based on the detection of toxic urine metabolites, including the principal metabolite, mono-(2-ethylhexyl) phthalate (MEHP) [14-16]. Lastly, we explore a plausible mechanism associated with idiopathic low-renin hypertension in premature infants by evaluating for MEHPinhibited $11 \beta$-HSD2 and consequential activation of mineralocorticoid receptor pathways.

\section{Methods}

A prospective observational study was conducted at two medical centers with approvals from the Institutional Review Boards and informed consent given for all participants. We recorded DEHP exposures and blood pressures in premature infants from birth for at least 6 months or until resolution of hypertension off medication occurred, whichever came last. Based on our prior study, we would expect no new cases of idiopathic hypertension after 6 months PMA, nor would we anticipate the ongoing need for anti-hypertensive medications beyond 8 months PMA [12]. Inclusion and exclusion criteria (Table 1) were designed to avoid bias related to clinical factors that might affect blood pressure. Study funding limited initial enrollment to 20 subjects, after which an analysis of the data was performed and presented herein. Two subjects were recruited monthly into the study until enrollment was complete. Although there were perhaps as many as 600 total potential premature infants to enroll, only $2-4$ met the study criteria at each of our bi-monthly recruiting days. The infants with the lowest gestational age were approached first to minimize the number of outpatient visits required.

The principal statistical comparisons between normotensive and hypertensive groups for DEHP exposures, blood pressure, and diagnostics were planned to be performed at the time of diagnosis of hypertension (and a corresponding PMA for normotensive infants). The diagnosis of hypertension was anticipated to be near 40 weeks PMA based on our prior work [12].

\section{Study procedures}

Table 2 describes the timeline and plan for study procedures. At enrollment, birthweight, gestational age at birth, and receipt of antenatal steroids were recorded, as these factors have been shown to be associated with (but not causative for) hypertension in premature infants [17-21]. Urine for sodium channel expression (described below) and a one-time urine sample for urine phthalate metabolites were the only diagnostic tests obtained at enrollment. The presence of DEHP exposure (defined below) for the $48 \mathrm{~h}$ prior to this one-time urine sampling was recorded since humans excrete DEHP into urine 12 to $48 \mathrm{~h}$ after DEHP exposure [22]. The presence of any DEHP exposure during the 48-h window was compared with the presence of any DEHP urine metabolites, including MEHP and two oxidative metabolites: mono-(2-ethyl-5hydroxyhexyl) phthalate (MEHHP) and mono-(2-ethyl-5oxohexyl) phthalate (MEOHP). To include the measurements of MEHHP and MEOHP results in an increase in the detection sensitivity for DEHP exposure as compared to MEHP measurement alone [3], urine phthalate metabolites were measured in a commercial lab using high performance liquid chromatography with tandem mass spectrometry (LC-MS/MS). 
Table 1 Inclusion and exclusion criteria for study

\begin{tabular}{|c|c|c|}
\hline Inclusion criteria & Exclusion criteria & Additional exclusion criteria at time of diagnosis of hypertension \\
\hline Gestation age $<37$ weeks & Secondary hypertension ${ }^{\mathrm{a}}$ & Receiving IV fluid or sodium supplementation \\
\hline Subject resides within 90 miles of center & Chronic kidney disease — any stage & Receiving diuretic or sympathomimetic agents \\
\hline \multirow[t]{2}{*}{ Age 2 weeks or less at entry ${ }^{\mathrm{b}}$} & Congenital renal abnormality ${ }^{\mathrm{c}}$ & Acute kidney injury ${ }^{\mathrm{d}}$ \\
\hline & Foster care, or surrogate birth & Patent ductus arteriosus (current) \\
\hline
\end{tabular}

Between enrollment and the time of the principal comparison (at hypertension diagnosis), every other week systolic blood pressure (SBP) and all DEHP exposures (defined below) were recorded. Urinary exosome expression of the epithelial sodium channel $(\mathrm{ENaC})$ and phosphorylated (activated) sodium chloride cotransporter (pNCC) were obtained every 4 weeks. These two sodium channels are upregulated by activation of the mineralocorticoid receptor (MR) $[23,24]$. The procedure for isolation of urinary exosomes was described by Van der Lubbe et al., with a modification such that urine and reagent volumes were scaled down by twothirds [25]. Activities were measured with Western Blot methodology using antibodies to the gamma subunit of $\mathrm{ENaC}$, pNCC, and CD9 (a marker of exosome material), and were expressed as a ratio of transporter expression with CD9 expression. Concise methods for exosome preparation and Western analysis are available in the Appendix.

Diagnostic tests reflecting sodium metabolism were obtained at the time of diagnosis of hypertension or at 40 weeks PMA for normotensive patients - the principal comparison timeframe. These tests, which were all obtained prior to initiating anti-hypertensive therapy (for hypertensive infants), were as follows: serum sodium, potassium, creatinine, aldosterone, plasma renin activity (PRA), ENaC, pNCC, and urine cortisol-to-cortisone ratio, a surrogate for $11 \beta$-HSD2 activity. Hypertensive and normotensive groups were compared for these tests as well as for blood pressure and DEHP exposures.

\section{Quantitation of DEHP exposures}

Equipment was evaluated for the presence of DEHP at both hospitals based on product labeling.

Di-(2-ethylhexyl) pthalate was found in selected IV fluid bags (but not in parenteral nutrition bags) and in most respiratory-related tubing (except low-flow nasal cannulas). Since product labels list the presence but not the amount of DEHP in the product, cumulative exposures to DEHP were quantified in aggregate by the following methodology: The volume $(\mathrm{mL})$ of IV fluid administered to the infant from DEHP-containing IV bags quantified the IV exposure and respiratory tubing exposure was quantified by the number of

Table 2 Timing of study procedures

\begin{tabular}{|c|c|c|c|c|}
\hline $\begin{array}{l}\text { Study Enrollment } \\
\text { First month of life }\end{array}$ & $\begin{array}{l}\text { Monitoring visits } \\
\text { Every } 4 \text { weeks from } \\
\text { enrollment through } \\
4 \text { months }\end{array}$ & $\begin{array}{l}\text { Main Comparison } \\
\text { At diagnosis of hypertension or } \\
40 \text { weeks PMA (if hypertensive) }\end{array}$ & $\begin{array}{l}\text { Post-hypertension visits } \\
\text { Every four weeks after } \\
\text { diagnosis of hypertension }\end{array}$ & $\begin{array}{l}\text { Study End } \\
\text { Resolution of } \\
\text { hypertension-at least } \\
\text { six months of age }\end{array}$ \\
\hline SBP index & SBP index ${ }^{a}$ & SBP index & SBP index & SBP index \\
\hline Urinary $\mathrm{ENaC}, \mathrm{pNCC}$ & Urinary $\mathrm{ENaC}, \mathrm{pNCC}$ & $\begin{array}{l}\text { U. ENaC, pNCC, } \\
\text { cortisol/cortisone }\end{array}$ & Urinary $\mathrm{ENaC}$, pNCC & $\begin{array}{l}\text { U. ENaC, pNCC, } \\
\text { cortisol/cortisone }\end{array}$ \\
\hline $\begin{array}{l}\text { Informed consent, demographics } \\
\text { and risk factors for hypertension }\end{array}$ & $\begin{array}{l}\text { Hypertension } \\
\text { determination }\end{array}$ & $\begin{array}{l}\text { PRA, serum sodium, potassium, } \\
\text { creatinine, and aldosterone }\end{array}$ & $\begin{array}{l}\text { Resolution of } \\
\text { hypertension } \\
\text { determination }\end{array}$ & $\begin{array}{l}\text { Resolution of } \\
\text { hypertension } \\
\text { determination }\end{array}$ \\
\hline $\begin{array}{l}\text { Urine phthalate analysis } \\
\text { (one-time) }\end{array}$ & Interval DEHP exposures & $\begin{array}{l}\text { Cumulative DEHP } \\
\text { exposures from birth }\end{array}$ & & Medication history \\
\hline Inclusion/exclusion & & Inclusion/exclusion & & \\
\hline
\end{tabular}

$P M A$ postmenstrual age, $E N a C$ epithelial sodium channel, $p N C C$ phosphorylated (activated) sodium chloride cotransporter, $D E H P$ di-(2-ethylhexyl phthalate), $S B P$ systolic blood pressure, $P R A$ plasma renin activity

${ }^{\text {a }} \mathrm{SBP}$ index (SBP/SBP 95th percentile) was determined at each visit and every 2 weeks for inpatients 
days the patient was connected to any respiratory tubing containing DEHP.

\section{Blood pressure measurement and cohort assignment}

Systolic blood pressure was measured by nurses using the oscillometric method for infants while in the NICU and by a single experienced physician using the auscultatory method on the right arm when seen in the outpatient clinical setting. The two oscillometric devices used were from Philips Medical Systems, BG Eindhoven, The Netherlands, and from SpaceLabs Inc., Redmond, Washington, U.S.A. For these blood pressure measurements, there was no specification as to which extremity was used for testing. Bedside, nurses were trained in the use of appropriate-sized blood pressure cuffs. Diastolic blood pressures were not used due to reported concern with accuracy of auscultatory diastolic blood pressure measurement in this age group [12]. Outpatient visits occurred every 4 weeks through 4 months of age (or resolution of hypertension), with a final visit at resolution of hypertension but no earlier than 6 months of age. If SBP exceeded the gestational age-adjusted 95 th percentile, the infant was asked to return within 7 days for a blood pressure recheck.

Assignment to either the hypertensive or normotensive cohort was based on blood pressure criteria. Infants met the criteria for inclusion in the hypertensive group if their mean daily SBP (3 or more measurements per day) exceeded the 95th percentile for at least three sequential days while in the NICU or three sequential visits for outpatients. The "day of diagnosis" for hypertensive patients was set as the first date that the diagnosis of hypertension was recorded in the medical record. For three cases where the "day of diagnosis" of hypertension was not clearly established in the medical record, the "day of diagnosis" was set as the date the patient first met the above blood pressure criteria. The SBP 95th percentiles used for this study originated from the normative data compiled by Dionne et al., which provides blood pressure norms adjusted by PMA for premature infants [26]. Because SBP varies greatly with PMA, SBP index (SBP/SBP 95th percentile) was calculated and used to represent systolic blood pressure relative to the PMA-adjusted 95th percentile for SBP.

\section{Statistical analysis}

A non-parametric, two-sided Wilcoxon rank sum test compared differences between the normotensive and hypertensive groups for continuous variables. Continuity correction was applied when needed. Pearson's chi-squared testing was used to compare categorical variables. When expected values for chi-squared testing were $<5$, an N-1Chi-squared test was used [27]. Correlations between DEHP exposures and risk factors of neonatal hypertension were calculated using a Pearson's correlation. Statistical analyses for this report were performed using $\mathrm{R}$ version 3.3.2 and Stata version 14.2 (R Core Team 2016; Stata Corp. 2015).
We examined the relationship between cumulative DEHP exposures and the SBP index on the day of diagnosis for hypertensive infants and at the 40 weeks PMA visit for normotensive infants using linear regressions. First, we modeled cumulative IV DEHP and respiratory DEHP exposures. Next, a 4-step mediation analysis was performed to understand whether the relationship between IV DEHP exposure and SBP index is mediated by the urine cortisol-to-cortisone ratio. If the magnitude of the coefficient greatly decreased when the cortisol-to-cortisone ratio was added to the model, one would conclude that the exposure and cortisol-to-cortisone ratios were inter-related [28]. Finally, to test the hypothesis that activation of the MR was the putative mechanism of blood pressure elevations seen in study infants, we performed linear regression between expression of the two MR-regulated renal sodium channel transporters, ( $\mathrm{ENaC}$ and $\mathrm{pNCC})$, and SBP index at the same principal comparison timeframe.

\section{Results}

Of the 26 patients approached for participation, 20 enrolled in the study. One patient unexpectedly died in the first month of life for reasons unrelated to blood pressure and another failed to return for study visits prior to the 40-week PMA visit. This left 18 patients available for analysis. No secondary hypertension occurred in this cohort, and no other patients met exclusion criteria.

Nine patients developed hypertension at a mean PMA of $40.6 \pm 3.4$ weeks. Three of these nine fulfilled criteria and began treatment for hypertension while still in the NICU. Two cases experienced mild hypertension while in the NICU and did not receive anti-hypertensive medication. Four of these nine patients met hypertensive criteria as an outpatient. Only three of these four received anti-hypertensive therapy. The duration of

Fig. 1 a. Systolic blood pressure index and DEHP exposures in normotensive (cyan) versus hypertensive infants (magenta) are shown in 2week intervals from 28 weeks postmenstrual age to the last study visit (approximately 8 months of age). Individual patient 2-week interval DEHP exposures are shown for both intravenous (open circles) and respiratoryrelated DEHP exposures (solid circles). Each point represents the timespecific SBP index of an individual subject, with the size of the point or circle adjusted according to the magnitude of the exposure. Since the relationship between IV and respiratory DEHP exposures is unknown, the scales used for the two different DEHP exposure types are not related. Yellow asterisks within the magenta line mark the individual start of spironolactone treatment for the six treated hypertensive patients. Significant differences in SBP index between groups are noted on the $\mathrm{X}$ axis as follows: ${ }^{*} p<0.05$, $* * p<0.001$. b. SBP index (lines) from a and mean ENaC activity (columns) in normotensive (cyan) versus hypertensive infants (magenta) is shown in 2week intervals from 28 weeks PMA to the last study visit. c. SBP index (lines) from a and mean pNCC activity (columns) in normotensive (cyan) versus hypertensive infants (magenta) from 28 weeks PMA to the last study visit. SBP, systolic blood pressure; PMA, postmenstrual age; DEHP (di-(2ethylhexyl) phthalate; $\mathrm{ENaC}$, epithelial sodium channel; pNCC, phosphorylated (activated) sodium chloride cotransporter 
treatment for the six infants treated for hypertension was $14.2 \pm$ 4.9 weeks. Hypertension only lasted $2.6 \pm 0.7$ weeks in the three untreated hypertensive infants. All treated infants received spironolactone monotherapy with SBP successfully dropping below the adjusted 95th percentile within 1 week. Spironolactone in treated patients was discontinued successfully
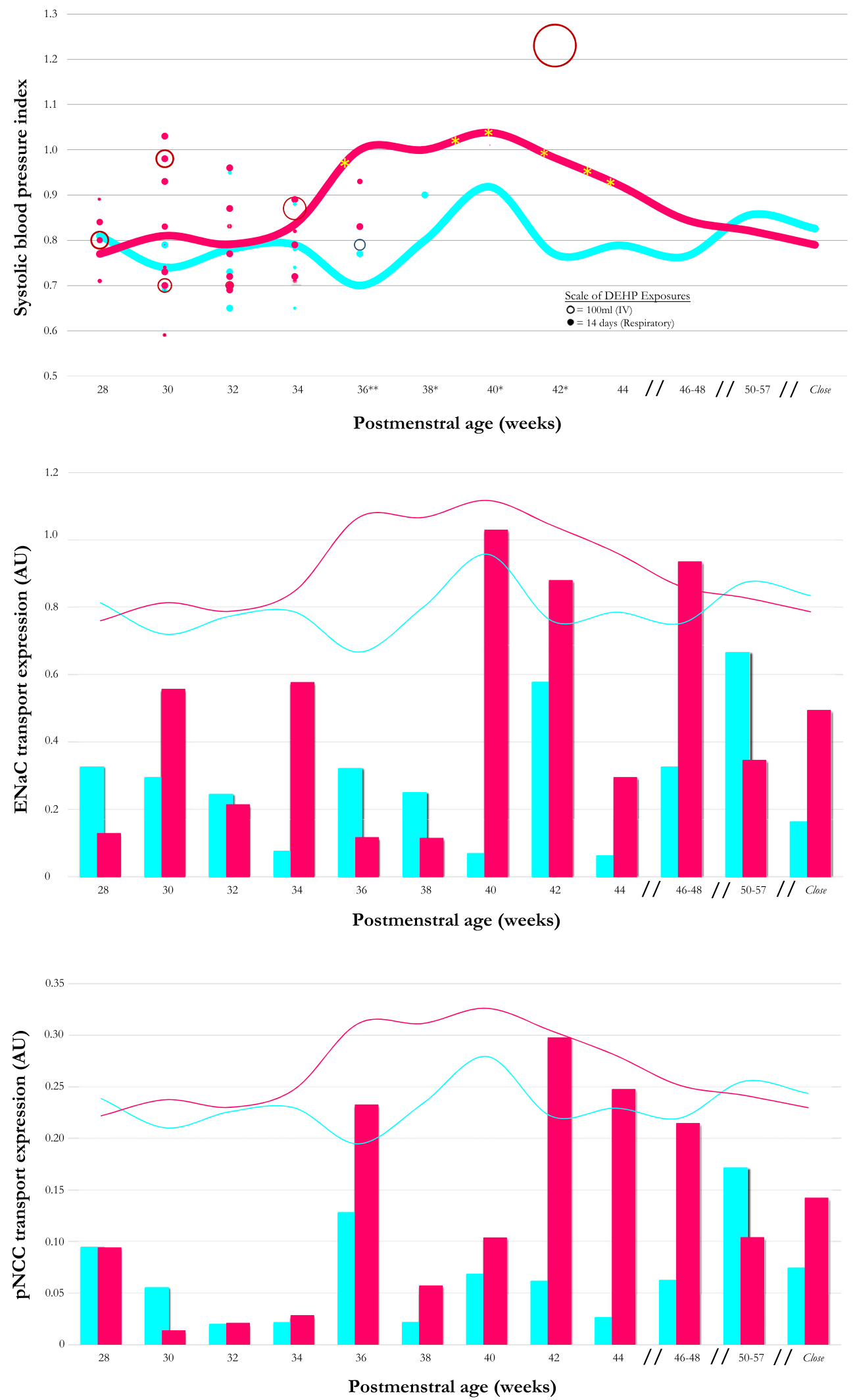
between 48 and 64 weeks PMA. All patients were off treatment at the study's end, with normal blood pressure not different from normotensive patients $(p<0.05)$.

Figure 1 provides a visual depiction of SBP index, intravenous, and respiratory-related DEHP exposures, and sodium transporter expression throughout the study period. Figure 1a depicts the average SBP index in hypertensive versus normotensive patients in relation to the type of DEHP exposure, whether respiratory or intravenous. On average, SBP for the hypertensive group did not increase until after 34 weeks PMA, with the SBP index peaking at 40 weeks PMA. Average group SBP normalized as infants began treatment with spironolactone. Normotensive patients showed a blunted peak of SBP index at 40 weeks PMA, but did not achieve levels consistent with systolic hypertension. Most exposures to either intravenous or respiratory DEHP in hypertensive patients occurred early in life, beginning before any rise in SBP index, mostly complete by 36 weeks PMA. The median time between the subjects' last DEHP exposure and diagnosis of hypertension was 3.57 weeks. Figure $1 \mathrm{~b}$ and $\mathrm{c}$ show the urinary exosomal expression of $\mathrm{ENaC}$ and $\mathrm{pNCC}$ for hypertensive and normotensive groups reported in 2-week intervals of postmenstrual age. Increased $\mathrm{ENaC}$ expression in the hypertensive group coincided with the peak SBP index around 40 weeks PMA. More interestingly, pNCC urinary exosomal expression in the hypertensive group was similar to that in the normotensive group through 34 weeks PMA. After 34 weeks PMA, pNCC expression was consistently higher in the hypertensive group compared to the normotensive group for the time when SBP index was higher in the hypertensive group.

Next, we move from the overview to our principal statistical comparison between the hypertensive group (at the diagnosis of hypertension), and the normotensive group (at 40 weeks PMA) for demographics, DEHP exposures, and risk factors for hypertension, which are shown in Table 3. Both intravenous and respiratory cumulative DEHP exposures were greater in the hypertensive as compared to the normotensive group ( $p=0.029, p=0.041$ respectively). Individual patient data for exposures and onset of hypertension are available in the Appendix. Gender, race, birthweight, receipt of antenatal steroids, and diagnosis of chronic lung disease did not differ significantly between the groups. Gestational age was lower in the hypertensive group, $(p=0.012)$. In addition, the length of stay for the initial NICU hospitalization was longer in the hypertensive group $(p=0.027)$.

All hypertensive patients had PRA levels that were below $2.0 \mathrm{ng} / \mathrm{mL} / \mathrm{h}$, compared to two of nine normotensive infants $(p=0.003)$. The common lower limit of normal for PRA in children is $2.0 \mathrm{ng} / \mathrm{mL} / \mathrm{h}$. At the 40 -week PMA principal comparison point, urinary cortisol-to-cortisone ratios were significantly higher in hypertensive infants as compared with normotensive infants $(p=0.02)$. Both $\mathrm{ENaC}$ and $\mathrm{pNCC}$, markers of MR activation, were significantly higher in hypertensive patients $(p=0.042, p=0.031)$.

Correlations between exposures and previously reported risk factors of hypertension in premature infants are shown in Table 4. Gestational age, birthweight, and length of stay are all correlated with cumulative respiratory DEHP exposure but not with IV DEHP exposure. Linear regression analysis demonstrated that SBP index at the principal comparison (onset of hypertension) was associated with cumulative IV DEHP exposure, but not respiratory DEHP exposure (Table 5). The urinary cortisol-to-cortisone ratio was predicted by intravenous DEHP exposure, and was highly predictive of SBP index. Figure 2 shows the close relationship between urinary cortisol-to-cortisone ratio and SBP index at onset of hypertension (or 40 weeks PMA for normotensive infants). In a multivariable model including IV DEHP exposure and the cortisol-to-cortisone ratio, the magnitude of the IV DEHP exposures effect on SBP index attenuates and approaches zeroevidence that the urinary cortisol-to-cortisone ratio mediates the relationship between DEHP exposure and SBP index (Table 6). Finally, both pNCC and $\mathrm{ENaC}$ were also predictive of SBP index at the diagnosis of hypertension (or 40 weeks PMA for normotensive infants) (Table 7).

The results for the DEHP urine metabolite testing are shown in Table 8. One sample was obtained in 14 of 20 enrolled infants - all at the study initiation visit (when blood pressure was normal in all). DEHP metabolites were present for six of ten infants with DEHP exposures documented during this $48 \mathrm{~h}$ window pre-sample. By N-1 Chi-square analysis, the presence of DEHP metabolites was related to the prior 48$\mathrm{h}$ IV DEHP exposure $(p=0.030)$, as well as prior respiratory DEHP exposure $(p=0.033)$. In contrast, when no DEHP exposure occurred during this 2-day period, no patient was found with detectable urinary DEHP metabolites.

\section{Discussion}

This study presents novel evidence that DEHP exposure is associated with systolic blood pressure increases in premature infants, including hypertension with larger exposures. The case for intravenous DEHP exposure increasing blood pressure is particularly strong given the following four findings: the linear relationship with SBP index, mediation of this relationship by the cortisol-to-cortisone ratio, and mechanistic links to MR activation.

The relationship between respiratory DEHP exposure and SBP is more complex. Although associated with hypertension in the studied cohort, no linear relationship was observed between respiratory DEHP exposure and SBP index, at least at the time of the diagnosis of hypertension. The inability to quantify respiratory DEHP exposure with precision confounded the ability to determine 
Table 3 Characteristics and comparisons between hypertensive and normotensive groups

\begin{tabular}{|c|c|c|c|}
\hline & $\begin{array}{l}\text { Hypertensive group } \\
n=9\end{array}$ & $\begin{array}{l}\text { Normotensive group } \\
n=9\end{array}$ & $p$ value \\
\hline \multicolumn{4}{|l|}{ Clinical characteristics } \\
\hline Gender: male (\#) & 4 & 7 & $0.136^{\mathrm{a}}$ \\
\hline Race: White, non-Hispanic (\#) & 7 & 6 & $0.492^{\mathrm{b}}$ \\
\hline Antenatal steroids received (\#) & 9 & 8 & $1.000^{\mathrm{b}}$ \\
\hline Chronic lung disease (\#) & 2 & 1 & $0.515^{\mathrm{a}}$ \\
\hline Birth weight $(\mathrm{kg})$ & $1.21 \pm 0.64$ & $1.72 \pm 0.69$ & $0.136^{\mathrm{d}}$ \\
\hline Gestational age at birth (weeks) & $28.5 \pm 2.2$ & $32.6 \pm 2.9$ & $0.012^{\mathrm{c}}$ \\
\hline Length of stay in neonatal unit (days) ${ }^{g}$ & $22(36)$ & $91(27)$ & $0.027^{\mathrm{d}}$ \\
\hline \multicolumn{4}{|l|}{ Comparisons } \\
\hline \multicolumn{4}{|l|}{ Between groups at onset of hypertension ( 40 weeks CGA for normotensive patients) } \\
\hline Time frame by CGA of comparison (weeks) & $40.6 \pm 3.4$ & $40.3 \pm 3.3$ & $0.930^{\mathrm{c}}$ \\
\hline Cumulative IV fluid DEHP exposure from birth to main comparison $(\mathrm{mL})^{\mathrm{g}}$ & $201.3(291.7)$ & $0(0)$ & $0.029^{\mathrm{c}}$ \\
\hline Cumulative respiratory DEHP exposure from birth to main comparison (days) ${ }^{\mathrm{e}, \mathrm{g}}$ & $45(38)$ & $7(7)$ & $0.041^{\mathrm{c}}$ \\
\hline SBP index (percentage of age-adjusted 95th percentile) & $1.11 \pm 0.10$ & $0.86 \pm 0.08$ & $<0.001^{\mathrm{d}}$ \\
\hline Plasma renin activity below $2.0 \mathrm{ng} / \mathrm{mL} / \mathrm{h}(\#)$ & 9 & 2 & $0.003^{\mathrm{a}}$ \\
\hline Serum sodium (meq/L) & $138.9 \pm 2.8$ & $140.0 \pm 2.6$ & $0.447^{\mathrm{d}}$ \\
\hline Serum potassium $(\mathrm{meq} / \mathrm{L})$ & $5.2 \pm 0.6$ & $5.3 \pm 1.0$ & $0.781^{\mathrm{d}}$ \\
\hline Serum creatinine $(\mathrm{mg} / \mathrm{dL})$ & $0.27 \pm 0.06$ & $0.29 \pm 0.05$ & $0.576^{\mathrm{d}}$ \\
\hline Serum aldosterone $(\mathrm{ng} / \mathrm{dL})^{\mathrm{g}}$ & $55.4(31.8)$ & $20.9(25.3)$ & $0.164^{\mathrm{d}}$ \\
\hline Urine cortisol-to-cortisone ratio & $0.07 \pm 0.02$ & $0.03 \pm 0.01$ & $0.020^{\mathrm{d}}$ \\
\hline pNCC protein expression ${ }^{\mathrm{f}, \mathrm{g}}$ & $0.402(0.127)$ & $0.086(0.105)$ & $0.042^{\mathrm{d}}$ \\
\hline $\mathrm{ENaC}$ protein expression ${ }^{\mathrm{f}, \mathrm{g}}$ & $0.723(0.316)$ & $0.279(0.243)$ & $0.031^{\mathrm{d}}$ \\
\hline \multicolumn{4}{|l|}{ Between groups at study close } \\
\hline Time frame by postnatal (chronologic) age (weeks) & $34.0 \pm 8.4$ & $36.5 \pm 10.3$ & $0.401^{\mathrm{d}}$ \\
\hline Systolic blood pressure (mmHg) & $83.22 \pm 6.87$ & $86.78 \pm 4.47$ & $0.211^{\mathrm{d}}$ \\
\hline
\end{tabular}

Continuous variables are shown as mean $( \pm \mathrm{SD})$; $C G A$ corrected gestational age, $P D A$ patent ductus arteriosus, $D E H P$ di-(2-ethylhexyl) phthalate, $S B P$ systolic blood pressure, $E N a C$ epithelial sodium channel, $p N C C$ phosphorylated (activated) sodium chloride cotransporter

${ }^{a} \mathrm{~N}-1$ chi-squared test

${ }^{\mathrm{b}}$ Pearson's chi-squared test with Yates's continuity correction

${ }^{\mathrm{c}}$ Wilcoxon rank sum test with continuity correction

${ }^{\mathrm{d}}$ Wilcoxon rank sum test

${ }^{\mathrm{e}}$ This variable includes days of mechanical ventilation, CPAP, high-flow nasal cannula, non-invasive positive pressure ventilation, but not low-flow nasal cannula

${ }^{\mathrm{f}}$ Sodium channel transport expression is expressed as activity per CD9 activity

${ }^{\mathrm{g}}$ Data are presented as median (IQR) due to their non-normal distribution

such a relationship. In addition, by virtue of the presence of DEHP in most respiratory equipment, respiratory DEHP exposure remains integrally linked with chronic

Table 4 Pearson's correlation coefficients for relationships between DEHP exposures and risk factors of hypertension in premature infants

\begin{tabular}{lllll}
\hline & IV DEHP & $p$ & Respiratory DEHP & $p$ \\
\hline Gestational age & -0.135 & 0.59 & -0.865 & $<0.001$ \\
Birthweight & 0.047 & 0.85 & -0.767 & $<0.001$ \\
Length of stay & 0.134 & 0.59 & 0.849 & $<0.001$ \\
\hline
\end{tabular}

lung disease and other markers of prematurity. This observation raises the question whether respiratory DEHP has a causal role in hypertension, or is merely just another marker of chronic lung disease and other risk factors related to extreme prematurity. A recent report of Stroustrup et al. showing premature infants exposed to respiratory support had significantly higher levels of DEHP urine metabolites compared to unexposed infants suggests the need for an in-depth look at the impact of respiratory DEHP on neonatal blood pressure [5].

The delay between DEHP exposures and peak blood pressure is notable, especially for intravenous DEHP exposures. 
Table 5 Linear model results for the relationship between DEHP exposures and systolic blood pressure (SBP) index at the diagnosis of hypertension (or 40 weeks postmenstrual age for normotensive infants)

\begin{tabular}{lllll}
\hline Independent variable & Dependent variable & $\beta$ coefficient & (95\% confidence interval) & $p$ \\
\hline Univariate model & & & \\
IV DEHP exposure (mL) & SBP index & 0.0004 & $0.0001-0.0008$ \\
Respiratory DEHP exposure (days) & SBP index & 0.0007 & $-0.0020-0.0034$ \\
\hline
\end{tabular}

$D E H P$ di-(2-ethylhexyl) phthalate, $I V$ intravenous, $S B P$ systolic blood pressure

One possible explanation for the apparent delayed effect was proposed by Martinez-Arguelles who offered that DEHP may act through a "second hit" phenomenon with initial latency [29]. Immaturity of sodium retention mechanisms of the very premature kidney is another explanation for this delay [30, 31]. The distal nephron may be unresponsive to MR stimuli until maturation of sodium transport function has occurred. Two observations support this explanation: ENaC expression in urinary exosomes, as an indirect measure of channel expression and activity, was not consistently elevated in hypertensive patients relative to controls until 40 weeks PMA; and, perhaps more significantly, pNCC was not elevated over the normotensive cohort prior to 36 weeks PMA, but manifested a higher expression thereafter until resolution of hypertension. This presumed tubular immaturity in the sodium channel and transporter expression may be analogous to that seen for neonatal potassium excretion, with a paucity of flow-dependent conducting potassium channels early in life [32].

The urine metabolite results suggest that both IV and respiratory DEHP exposures yield systemic absorption and formation of urinary MEHP. The data is not robust enough to estimate the magnitude or variability of DEHP that might leach from the various devices, nor can we predict how different respiratory DEHP exposures compare with each other or with
Fig. 2 Linear regression for urinary cortisol-to-cortisone ratio as a predictor of systolic blood pressure (SBP) index at the time of diagnosis of hypertension (or 40 weeks postmenstrual age for normotensive infants $(p<0.001)$. The SBP index is the ratio of SBB with the 95th percentile for SBP adjusted for postmenstrual age. Depicted is the linear regression. SBP, systolic blood pressure

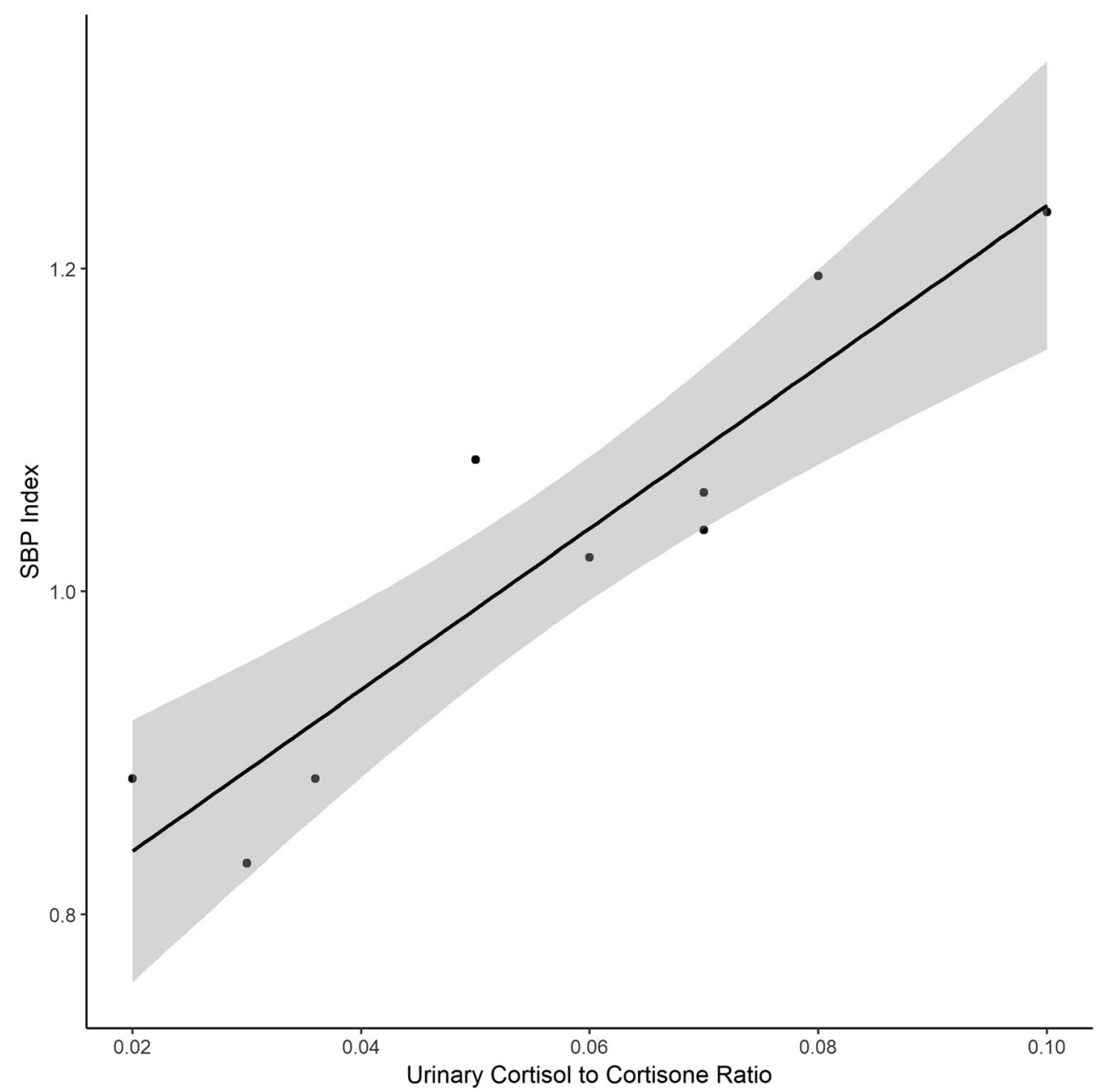


Table 6 Mediation analysis to evaluate urine cortisol-to-cortisone ratio as a mediator of the IV DEHP effect on systolic blood pressure index at the diagnosis of hypertension (or 40 weeks PMA for normotensive infants). The magnitude of the coefficient drops toward zero when urine cortisol-to-cortisone ratio is added to the model, suggesting the latter ratio mediates the effect of IV DEHP exposure on SBP index

\begin{tabular}{llll}
\hline Independent variable & Dependent variable & $\beta$ coefficient & $(95 \%$ confidence interval) \\
\hline Univariate model & & & \\
IV DEHP exposure $(\mathrm{mL})$ & Cortisol-to-cortisone ratio & 0.0001 & $1.64 \mathrm{e}-06-0.0001$ \\
Urine cortisol-to-cortisone ratio & SBP index & 5.0389 & $3.2058-6.8721$ \\
IV DEHP exposure (mL) & SBP index & 0.0004 & $0.0001-0.0008$ \\
Multivariable model including IV DEHP exposure and cortisol-to-cortisone ratio & & 0.046 \\
IV DEHP exposure (mL & SBP index & -0.0001 & $-0.0003-0.0002$ \\
Urine cortisol-to-cortisone ratio & SBP index & 5.4849 & $2.7859-9.1839$ \\
\hline
\end{tabular}

$D E H P$ di-(2-ethylhexyl) phthalate, $S B P$ systolic blood pressure, $I V$ intravenous, $P M A$ postmenstrual age

IV DEHP exposures concerning the effect on blood pressure. Previous studies have shown a systemic transfer from DEHPcontaining IV bags and PVC tubes into the bloodstream or other tissues [33, 34]. DEHP does not form covalent bonds to the PVC polymer, allowing DEHP to leach out of the PVC polymer matrix. The rate of DEHP leaching is influenced by lipophilic qualities of the fluid in contact, temperature, contact time, contact surface area, and fluid flow rate [33, 35-39]. There are estimates of DEHP leaching rates for endotracheal tubes [4], blood transfusions [40], lipid infusions [33], but not for specific PVC respiratory circuitry or IV fluid contained in bags with DEHP. One NICU unit, which labeled itself as "phthalate-free" signifying attempts to use DEHP-free equipment when available, still found detectable levels of MEHP in all of the 149 urine specimens tested [5].

Studies on mechanisms and effects of phthalate toxicity performed mostly on rats have examined both maternal and developing infant exposure [14-16, 29, 41]. Endocrine disruption effects, described as anti-androgenic, have been extensively reported from prenatal phthalate exposure and are summarized in a recent review [42]. Martinez-Arguelles et al. recently reviewed current knowledge of mechanisms mediating endocrine disruption from in-utero DEHP exposure that include alterations in adrenal steroidogenesis as well as evidence of epigenetic changes in rats leading to disease later in life [29, 41]. Studies of direct DEHP exposure in children have shown evidence of both endocrine and non-endocrine toxicity and have been reviewed elsewhere [14, 43, 44].
Aside from Zhao's work, there are few other studies suggesting mechanisms that might explain how phthalate exposure could increase blood pressure. There is evidence that phthalates may cause oxidative stress $[45,46]$. Kambia et al. treated infants and children with hyperalimentation given through PVC tubing containing DEHP and found that plasma DEHP levels correlated with malondialdehyde, a marker of free radical production [38]. Such oxidative stress may produce temporary changes in arterial tone, which increases blood pressure [47, 48]. Meanwhile, Zhao's work, which exposed human tissue to various phthalates, convincingly demonstrated that MEHP (but not DEHP) was a potent inhibitor of human $11 \beta-H S D 2$ at clinically relevant concentrations [11]. When $11 \beta$-HSD2 is inhibited by black licorice, or when it is deficient as a result of genetic mutations (apparent mineralocorticoid excess), the result is sodium retention and hypertension [49].

Analysis of our data strongly suggests the mechanism of hypertension in our study cohort includes activation of the mineralocorticoid receptor. Our demonstration of increased sodium channel and transporter expression predicting higher SBP index aligns with activation of the MR, for $\mathrm{ENaC}$ at the height of the SBP index difference, and for pNCC throughout the entire time frame of the difference in SBP index between the two groups (PMA weeks 36 to 42). The evidence showing that urinary cortisol-to-cortisone ratio mediates the effect of IV DEHP on the SBP index suggests that inhibition of $11 \beta$ HSD2 by DEHP metabolites leads to MR activation in our
Table 7 Linear regression model results for the relationship between sodium channel expression and SBP index at the diagnosis of hypertension (or 40 weeks postmenstrual age for normotensive infants)

\begin{tabular}{lllll}
\hline Independent variable & Dependent variable & $\beta$ coefficient & $(95 \%$ confidence interval $)$ & $p$ \\
\hline Univariate model & & & & \\
pNCC & SBP index & 0.7025 & $0.3115-1.0934$ & $0.003^{\mathrm{a}}$ \\
ENaC & SBP index & 0.1955 & $0.0205-0.3704$ & $0.032^{\mathrm{a}}$ \\
\hline
\end{tabular}

$D E H P$ di-(2-ethylhexyl) phthalate, $I V$ intravenous, $S B P$ systolic blood pressure, $p N C C$ phosphorylated (activated) sodium chloride cotransporter, $E N a C$ epithelial sodium channel ${ }^{\mathrm{a}} p<0.05$ 
Table 8 Presence or absence of urinary DEHP metabolites as detected from a single urine sample at study enrollment as compared with presence or absence of DEHP exposures during the prior $48 \mathrm{~h}$

\begin{tabular}{|c|c|c|c|c|c|}
\hline Subject $(\#)^{\mathrm{a}}$ & $\begin{array}{l}\text { Respiratory DEHP exposures } \\
\text { (days) }\end{array}$ & IV DEHP exposures (mL) & $\begin{array}{l}\text { MEHHP } \\
(\mathrm{ng} / \mathrm{mL}) \\
(\mathrm{mcg} / \mathrm{g} \text { creatinine })\end{array}$ & $\begin{array}{l}\text { MEOHP } \\
(\mathrm{ng} / \mathrm{mL}) \\
(\mathrm{mcg} / \mathrm{g} \text { creatinine })\end{array}$ & $\begin{array}{l}\text { MEHP } \\
(\mathrm{ng} / \mathrm{mL}) \\
(\mathrm{mcg} / \mathrm{g} \text { creatinine })\end{array}$ \\
\hline $\mathrm{N} 1^{\mathrm{d}}$ & 2 (bubble CPAP) & 0 & Assay failed & $\begin{array}{l}1600 \\
12,000\end{array}$ & $\begin{array}{l}650 \\
4600\end{array}$ \\
\hline $\mathrm{N} 2^{\mathrm{b}}$ & 0 & 0 & $<$ LOD & $<$ LOD & $<$ LOD \\
\hline $\mathrm{N} 3^{\mathrm{b}}$ & 0 & 0 & $<$ LOD & $<$ LOD & $<$ LOD \\
\hline $\mathrm{N} 4^{\mathrm{b}}$ & 0 & 0 & $<$ LOD & $<$ LOD & $<$ LOD \\
\hline $\mathrm{N} 5^{\mathrm{d}}$ & 2 (Hi-flow and ventilator) & 9 & Assay failed & $\begin{array}{l}<\mathrm{LOD} \\
\text { No urine creatinine }\end{array}$ & $\begin{array}{l}30 \\
\text { No urine creatinine }\end{array}$ \\
\hline $\mathrm{N} 6^{\mathrm{c}}$ & 2 (Hi-flow) & 0 & Assay failed & Assay failed & $<$ LOD \\
\hline $\mathrm{N} 7^{\mathrm{e}}$ & NA & NA & Not done & Not done & Not done \\
\hline $\mathrm{N} 8^{\mathrm{e}}$ & NA & NA & Not done & Not done & Not done \\
\hline $\mathrm{N} 9^{\mathrm{e}}$ & NA & NA & Not done & Not done & Not done \\
\hline $\mathrm{D} 1^{\mathrm{d}}$ & 2 (Ventilator) & 10 & Assay failed & $\begin{array}{l}69 \\
420\end{array}$ & $\begin{array}{l}32 \\
200\end{array}$ \\
\hline $\mathrm{D} 2^{\mathrm{e}}$ & NA & NA & Not done & Not done & Not done \\
\hline $\mathrm{H} 1^{\mathrm{e}}$ & NA & NA & Not done & Not done & Not done \\
\hline $\mathrm{H} 2^{\mathrm{d}}$ & 2 (NIPPV) & 0 & Assay failed & Assay failed & $\begin{array}{l}35 \\
240\end{array}$ \\
\hline $\mathrm{H} 3^{\mathrm{c}}$ & 2 (NIPPV) & 0 & $<$ LOD & $<$ LOD & $<$ LOD \\
\hline $\mathrm{H} 4^{\mathrm{b}}$ & 0 & 0 & $<$ LOD & $<$ LOD & $<\mathrm{LOD}$ \\
\hline $\mathrm{H} 5^{\mathrm{d}}$ & 2 (Hi-flow) & 49 & $\begin{array}{l}45 \\
400\end{array}$ & $\begin{array}{l}25 \\
220\end{array}$ & $<\mathrm{LOD}$ \\
\hline $\mathrm{H} 6^{\mathrm{c}}$ & 2 (mask CPAP) & 0 & $<$ LOD & $<$ LOD & $<$ LOD \\
\hline $\mathrm{H} 7^{\mathrm{c}}$ & 2 (mask CPAP) & 12 & $<$ LOD & $<$ LOD & $<$ LOD \\
\hline $\mathrm{H} 8^{\mathrm{d}}$ & 2 (NIPPV) & 24 & $\begin{array}{l}49 \\
400\end{array}$ & $\begin{array}{l}18 \\
150\end{array}$ & $<\mathrm{LOD}$ \\
\hline $\mathrm{H} 9^{\mathrm{e}}$ & NA & NA & Not done & Not done & Not done \\
\hline
\end{tabular}

$D E H P$ di-(2-ethylhexyl) phthalate, $M E H H P$ mono-(2-ethyl-5-hydroxyhexyl) phthalate, $M E O H P$ mono-(2-ethyl-5-oxohexyl) phthalate, $M E H P$ mono(2-ethylhexyl) phthalate, $C P A P$ continuous positive airway pressure, NIPPV non-invasive positive pressure ventilation, $L O D$ level of detection

${ }^{a}$ Urine samples (one each) were obtained on 14 subjects including 1 dropout (D), 6 normotensive $(\mathrm{N})$, and 8 hypertensive (H) subjects

${ }^{\mathrm{b}}$ Infants with no exposures and no detectable phthalate metabolites

${ }^{\mathrm{c}}$ Infants with DEHP exposures (3 respiratory and 1 combined) and no detectable phthalate metabolites

${ }^{\mathrm{d}}$ Infants with DEHP exposures (2 respiratory and 4 combined) who had detectable urine phthalate metabolites

${ }^{\mathrm{e}}$ Infants where no sampling was done

cohort, in keeping with the observation by Zhao on the effect of DEHP metabolites on 11 $\beta$-HSD2 [11].

The same clinical characteristics of low PRA, presentation around 35-40 weeks PMA, and an excellent response to spironolactone therapy - all seen in both our prior study [12] and in that of Al Awad et al. [13] - were also seen in this current study cohort. These similarities suggest that the mechanism of action found in this cohort may be generalizable to other premature infants with idiopathic or unexplained low-renin hypertension, with or without chronic lung disease.

Strengths of this study include its prospective design as well as urine metabolite data demonstrating DEHP transfer from medical devices to patients. Further, at least two analyses lend credence to the study's hypotheses: (1) the linear relationship between IV exposures and SBP difference and (2) the analysis showing this relationship was mediated by urine cortisol-to-cortisone ratio. Weaknesses of the study include the lack of precise quantitation of DEHP exposures from respiratory devices and the small sample size. With only 18 subjects for analysis, we were limited in the complexity of our model building and therefore tested only the main variables of interest, which were IV and respiratory exposures to DEHP, and the effect that exposures had on SBP index and the cortisol-to-cortisone ratio. Ideally, we would have been able to control for important variables such as gestational age or similar markers of extreme prematurity previously linked to hypertension in premature infants. 
In conclusion, both IV and respiratory DEHP exposures are greater in hypertensive patients as compared to normotensive patients. Cumulative IV DEHP exposure is predictive of SBP index, a relationship mediated via the cortisol-to-cortisone ratio. As DEHP is metabolized to MEHP, a known inhibitor of $11 \beta$ HSD2, a plausible mechanism of MR-mediated sodium retention, increased blood pressure, and low PRA is proposed. These results are provocative as to the potential role played by phthalates in idiopathic hypertension in premature infants. To determine the significance and safety of DEHP in respiratory tubing in regard to blood pressure effects, further studies are warranted.

Acknowledgements We thank D. Ellison for guidance and provision of rabbit polyclonal anti-pNCC antibodies, T. Cornwall for work determining sodium channel expression, and A. Stout for editing and graphics support.

Funding This study was funded by the Friends of Doernbecher Foundation with additional support by a gift from Ray and Margaret Thiercof in memory of Beth and Chrissy Thiercof.

\section{Compliance with ethical standards}

Conflict of interest The authors declare they have no conflict of interest.

Ethical approval All procedures performed in studies involving human participants were in accordance with the ethical standards of the institutional and/or national research committee and with the 1964 Helsinki declaration and its later amendments or comparable ethical standards.

Open Access This article is distributed under the terms of the Creative Commons Attribution 4.0 International License (http:// creativecommons.org/licenses/by/4.0/), which permits unrestricted use, distribution, and reproduction in any medium, provided you give appropriate credit to the original author(s) and the source, provide a link to the Creative Commons license, and indicate if changes were made.

\section{References}

1. Shea KM, American Academy of Pediatrics Committee on Environmental H (2003) Pediatric exposure and potential toxicity of phthalate plasticizers. Pediatrics 111(6 Pt 1):1467-1474

2. Green R, Hauser R, Calafat AM, Weuve J, Schettler T, Ringer S, Huttner $\mathrm{K}, \mathrm{Hu} \mathrm{H}$ (2005) Use of di(2-ethylhexyl) phthalatecontaining medical products and urinary levels of mono(2ethylhexyl) phthalate in neonatal intensive care unit infants. Environ Health Perspect 113(9):1222-1225

3. Weuve J, Sanchez BN, Calafat AM, Schettler T, Green RA, Hu H, Hauser R (2006) Exposure to phthalates in neonatal intensive care unit infants: urinary concentrations of monoesters and oxidative metabolites. Environ Health Perspect 114(9):1424-1431

4. Chiellini F, Ferri M, Latini G (2011) Physical-chemical assessment of di-(2-ethylhexyl)-phthalate leakage from poly(vinyl chloride) endotracheal tubes after application in high risk newborns. Int $\mathrm{J}$ Pharm 409(1-2):57-61. https://doi.org/10.1016/j.ijpharm.2011.02. 024

5. Stroustrup A, Bragg JB, Busgang SA, Andra SS, Curtin P, Spear EA, Just AC, Arora M, Gennings C (2018) Sources of clinically significant neonatal intensive care unit phthalate exposure. J Expo
Sci Environ Epidemiol. https://doi.org/10.1038/s41370-018-00692

6. Calafat AM, Needham LL, Silva MJ, Lambert G (2004) Exposure to di-(2-ethylhexyl) phthalate among premature neonates in a neonatal intensive care unit. Pediatrics 113(5):e429-e434

7. Trasande L, Sathyanarayana S, Spanier AJ, Trachtman H, Attina TM, Urbina EM (2013) Urinary phthalates are associated with higher blood pressure in childhood. J Pediatr 163(3):747-753 e741. https://doi.org/10.1016/j.jpeds.2013.03.072

8. Shiue I (2014) Higher urinary heavy metal, phthalate, and arsenic but not parabens concentrations in people with high blood pressure, U.S. NHANES, 2011-2012. Int J Environ Res Public Health 11(6): 5989-5999. https://doi.org/10.3390/ijerph110605989

9. Trasande L, Attina TM (2015) Association of exposure to di-2ethylhexylphthalate replacements with increased blood pressure in children and adolescents. Hypertension 66(2):301-308. https://doi. org/10.1161/HYPERTENSIONAHA.115.05603

10. Flynn JT, Kaelber DC, Baker-Smith CM, Blowey D, Carroll AE, Daniels SR, de Ferranti SD, Dionne JM, Falkner B, Flinn SK, Gidding SS, Goodwin C, Leu MG, Powers ME, Rea C, Samuels J, Simasek M, Thaker VV, Urbina EM, Subcommittee On S, Management Of High Blood Pressure In C (2017) Clinical practice guideline for screening and management of high blood pressure in children and adolescents. Pediatrics 140(3). https://doi.org/10.1542/ peds.2017-1904

11. Zhao B, Lian Q, Chu Y, Hardy DO, Li XK, Ge RS (2011) The inhibition of human and rat 11 beta-hydroxysteroid dehydrogenase 2 by perfluoroalkylated substances. J Steroid Biochem Mol Biol 125(1-2):143-147. https://doi.org/10.1016/j.jsbmb.2010.12.017

12. Jenkins RD, Aziz JK, Gievers LL, Mooers HM, Fino N, Rozansky DJ (2017) Characteristics of hypertension in premature infants with and without chronic lung disease: a long-term multi-center study. Pediatr Nephrol 32(11):2115-2124. https://doi.org/10.1007/ s00467-017-3722-4

13. Al Awad E, Yusuf K, Soraisham A, Obaid H, Sundaram A, Samedi V, Akierman A (2018) Transient hyperaldosteronism and neonatal hypertension: case series and literature review. J Clin Neonatol 7(3): 185-189. https://doi.org/10.4103/jcn.JCN_57_18

14. Phthalates and Cumulative Risk Assessment: The Tasks Ahead (2008). National Research Council of the National Academies, Washington (DC). https://doi.org/10.17226/12528

15. Toxicity Review of Di(2-ethylhexyl) Phthalate (DEHP) (2010) United States Consumer Product Safety Commission, Bethesda, MD

16. Opinion on the Safety of Medical Devices Containing DEHPPlasticized PVC or Other Plasticizers on Neonates and Other Groups Possibly at Risk (2008). Scientific Committee on Emerging and Newly Identified Health Risks (SCENIHR), Brussels, Belgium

17. Friedman AL, Hustead VA (1987) Hypertension in babies following discharge from a neonatal intensive care unit. A 3-year followup. Pediatr Nephrol 1(1):30-34

18. Singh HP, Hurley RM, Myers TF (1992) Neonatal hypertension. Incidence and risk factors. Am J Hypertens 5(2):51-55

19. Seliem WA, Falk MC, Shadbolt B, Kent AL (2007) Antenatal and postnatal risk factors for neonatal hypertension and infant followup. Pediatr Nephrol 22(12):2081-2087. https://doi.org/10.1007/ s00467-007-0603-2

20. Kent AL, Chaudhari T (2013) Determinants of neonatal blood pressure. Curr Hypertens Rep 15(5):426-432. https://doi.org/10.1007/ s11906-013-0375-y

21. Sahu R, Pannu H, Yu R, Shete S, Bricker JT, Gupta-Malhotra M (2013) Systemic hypertension requiring treatment in the neonatal intensive care unit. J Pediatr 163(1):84-88. https://doi.org/10.1016/ j.jpeds.2012.12.074 
22. Hoppin JA, Brock JW, Davis BJ, Baird DD (2002) Reproducibility of urinary phthalate metabolites in first morning urine samples. Environ Health Perspect 110(5):515-518

23. Moes AD, van der Lubbe N, Zietse R, Loffing J, Hoorn EJ (2014) The sodium chloride cotransporter SLC12A3: new roles in sodium, potassium, and blood pressure regulation. Pflugers Arch 466(1): 107-118. https://doi.org/10.1007/s00424-013-1407-9

24. Subramanya AR, Ellison DH (2014) Distal convoluted tubule. Clin J Am Soc Nephrol 9(12):2147-2163. https://doi.org/10.2215/CJN. 05920613

25. van der Lubbe N, Lim CH, Meima ME, van Veghel R, Rosenbaek LL, Mutig K, Danser AH, Fenton RA, Zietse R, Hoorn EJ (2012) Aldosterone does not require angiotensin II to activate NCC through a WNK4-SPAK-dependent pathway. Pflugers Arch 463(6):853-863. https://doi.org/10.1007/s00424-012-1104-0

26. Dionne JM, Abitbol CL, Flynn JT (2012) Hypertension in infancy: diagnosis, management and outcome. Pediatr Nephrol 27(1):17-32. https://doi.org/10.1007/s00467-010-1755-z

27. Campbell I (2007) Chi-squared and Fisher-Irwin tests of two-bytwo tables with small sample recommendations. Stat Med 26(19): 3661-3675. https://doi.org/10.1002/sim.2832

28. Baron RMKD (1986) The moderator-mediator variable distinction in social psychological research: conceptual, strategic, and statistical considerations. J Pers Soc Psychol 51(6):1173-1182

29. Martinez-Arguelles DB, Papadopoulos V (2015) Mechanisms mediating environmental chemical-induced endocrine disruption in the adrenal gland. Front Endocrinol (Lausanne) 6:29. https://doi.org/ 10.3389/fendo.2015.00029

30. Leslie GI, Barr PA, Gallery ED, Gyory AZ (1984) Role of renin and aldosterone in establishment of electrolyte balance in very low birthweight neonates. Aust Paediatr J 20(3):209-212

31. Bourchier D (2005) Plasma aldosterone levels in the 1st week of life in infants of less than 30 weeks gestation. Eur J Pediatr 164(3):141145. https://doi.org/10.1007/s00431-004-1572-0

32. Zhou H, Satlin LM (2004) Renal potassium handling in healthy and sick newborns. Semin Perinatol 28(2):103-111

33. Loff S, Kabs F, Subotic U, Schaible T, Reinecke F, Langbein M (2002) Kinetics of diethylhexyl-phthalate extraction from polyvinylchloride-infusion lines. JPEN J Parenter Enteral Nutr 26(5):305-309. https://doi.org/10.1177/0148607102026005305

34. Subotic U, Hannmann T, Kiss M, Brade J, Breitkopf K, Loff S (2007) Extraction of the plasticizers diethylhexylphthalate and polyadipate from polyvinylchloride nasogastric tubes through gastric juice and feeding solution. J Pediatr Gastroenterol Nutr 44(1): 71-76. https://doi.org/10.1097/01.mpg.0000237939.50791.4b

35. Peck CC, Odom DG, Albro PW, Jess DA, Barrett BB (1981) Effect of heat on the conversion of di-2-ethylhexyl phthalate to mono-2ethylhexyl phthalate in human plasma. Transfusion 21(2):163-166

36. Loff S, Subotic U, Reinicke F, Wischmann H, Brade J (2004) Extraction of di-ethylhexyl-phthalate from perfusion lines of various material, length and brand by lipid emulsions. J Pediatr Gastroenterol Nutr 39(4):341-345

37. Latini G, De Felice C, Del Vecchio A, Barducci A, Ferri M, Chiellini F (2009) Di-(2-ethylhexyl)phthalate leakage and color changes in endotracheal tubes after application in high-risk newborns. Neonatology 95(4):317-323. https://doi.org/10.1159/ 000181161
38. Kambia N, Dine T, Gressier B, Frimat B, Cazin JL, Luyckx M, Brunet C, Michaud L, Gottrand F (2011) Correlation between exposure to phthalates and concentrations of malondialdehyde in infants and children undergoing cyclic parenteral nutrition. JPEN J Parenter Enteral Nutr 35(3):395-401. https://doi.org/10.1177/ 0148607110381769

39. Bernard L, Cueff R, Breysse C, Decaudin B, Sautou V, Armed Study G (2015) Migrability of PVC plasticizers from medical devices into a simulant of infused solutions. Int J Pharm 485(1-2): 341-347. https://doi.org/10.1016/j.ijpharm.2015.03.030

40. Mallow EB, Fox MA (2014) Phthalates and critically ill neonates: device-related exposures and non-endocrine toxic risks. J Perinatol 34(12):892-897. https://doi.org/10.1038/jp.2014.157

41. Meltzer D, Martinez-Arguelles DB, Campioli E, Lee S, Papadopoulos V (2015) In utero exposure to the endocrine disruptor di(2-ethylhexyl) phthalate targets ovarian theca cells and steroidogenesis in the adult female rat. Reprod Toxicol 51: 47-56. https://doi.org/10.1016/j.reprotox.2014.12.005

42. Endocrine disrupting chemicals. (2012) Paper presented at the United Nations Environmental Program, Stockholm, Sweden

43. Swan SH (2008) Environmental phthalate exposure in relation to reproductive outcomes and other health endpoints in humans. Environ Res 108(2):177-184

44. Bustamante-Montes LP, Hernandez-Valero MA, Flores-Pimentel D, Garcia-Fabila M, Amaya-Chavez A, Barr DB, Borja-Aburto VH (2013) Prenatal exposure to phthalates is associated with decreased anogenital distance and penile size in male newborns. J Dev Orig Health Dis 4(4):300-306. https://doi.org/10.1017/ S2040174413000172

45. Hong YC, Park EY, Park MS, Ko JA, Oh SY, Kim H, Lee KH, Leem JH, Ha EH (2009) Community level exposure to chemicals and oxidative stress in adult population. Toxicol Lett 184(2):139 144. https://doi.org/10.1016/j.toxlet.2008.11.001

46. Ferguson KK, Loch-Caruso R, Meeker JD (2011) Urinary phthalate metabolites in relation to biomarkers of inflammation and oxidative stress: NHANES 1999-2006. Environ Res 111(5):718-726. https:// doi.org/10.1016/j.envres.2011.02.002

47. Alexander RW (1995) Theodore Cooper memorial lecture. Hypertension and the pathogenesis of atherosclerosis. Oxidative stress and the mediation of arterial inflammatory response: a new perspective. Hypertension 25(2):155-161

48. Ostrow V, Wu S, Aguilar A, Bonner R Jr, Suarez E, De Luca F (2011) Association between oxidative stress and masked hypertension in a multi-ethnic population of obese children and adolescents. J Pediatr 158(4):628-633 e621. https://doi.org/10.1016/j.jpeds. 2010.09.081

49. Walker BR, Edwards CR (1994) Licorice-induced hypertension and syndromes of apparent mineralocorticoid excess. Endocrinol Metab Clin N Am 23(2):359-377

Publisher's note Springer Nature remains neutral with regard to jurisdictional claims in published maps and institutional affiliations. 